Research paper

\title{
Ibrutinib-based therapy impaired neutrophils microbicidal activity in patients with chronic lymphocytic leukemia during the early phases of treatment
}

\author{
Alessandro Prezzo $^{\mathrm{a}, 1}$, Filomena Monica Cavaliere ${ }^{\mathrm{a}, 1}$, Caterina Bilotta ${ }^{\mathrm{a}}$, \\ Tancredi Massimo Pentimalli ${ }^{\mathrm{a}}$, Metello Iacobini ${ }^{\mathrm{b}}$, Laura Cesini ${ }^{\mathrm{c}}$, Robin Foà ${ }^{\mathrm{c}}$, \\ Francesca Romana Mauro ${ }^{c}$, Isabella Quinti ${ }^{\mathrm{a}, *}$ \\ ${ }^{a}$ Department of Molecular Medicine, Sapienza University, Viale Regina Elena, 291, 00161 Rome, Italy \\ ${ }^{\mathrm{b}}$ Department of Pediatrics, Sapienza University, Viale Regina Elena 324, 00161 Rome, Italy \\ ${ }^{\mathrm{c}}$ Hematology, Department of Translational and Precision Medicine, Sapienza University, Rome, Italy
}

\section{A R T I C L E I N F O}

\section{Keywords:}

CLL

Neutrophils

Oxidative burst

$\mathrm{NE}$

MPO

LF

\begin{abstract}
A B S T R A C T
Ibrutinib is a tyrosine kinase inhibitor used in the treatment of a variety of lymphoid malignancies, including chronic lymphocytic leukemia (CLL). Drugs inhibiting B-cell-receptor (BCR)-associated kinases, including BTK inhibitors, act on B cells and on a wide spectrum of tissues and cells, including innate immunity cells. Thus, alterations in the Bruton's tyrosine kinase (BTK) kinase function could lead to an impairment of innate immune cells functions and to an increased infectious risk in patients receiving BTK inhibitors. We analyzed in vivo neutrophils oxidative burst, neutrophils granules release and cytokine production in relapsed/refractory CLL patients treated over time with ibrutinib as single-agent. We observed a dramatic reduction of neutrophils oxidative burst, Fc gamma receptors ( $\mathrm{Fc} \gamma \mathrm{Rs}$ )-mediated degranulation and IL-8 plasma levels already after the first forty-eight hours of therapy with ibrutinib. However, ibrutinib treatment did not alter the surface expression of $\mathrm{CD} 11 \mathrm{~b}$ nor cytokine and proteinases release not mediated by Fc $\gamma$ Rs engagement. After three weeks, oxidative burst was still impaired, while degranulation and IL-8 levels were restored. In a group of CLL patients who survived for more than three years, all processes triggered by Fc $\gamma$ Rs completely recovered except the release of neutrophil elastase (NE) and IL-8. In conclusion, during the initial phases of ibrutinib therapy, the reduction of IL-8, NE, myeloperoxidase (MPO) levels and oxidative burst negatively impacted on mechanisms involved in neutrophils microbicidal activity.
\end{abstract}

\section{Introduction}

CLL, the most common hematologic malignancy in adults among western countries, is characterized by accumulation of mature clonal B lymphocytes in blood, lymph nodes and bone marrow [1-3].

CLL is characterized by several abnormalities in the adaptive and innate immune system associated with an increased risk of bacterial and fungal infections accountable for a high morbidity and mortality $[3,4]$. Several studies on CLL have described a reduction of complement proteins, an impair killing of non opsonized bacteria, a reduction in C5a-induced chemotaxis, a deficiency in myeloperoxidase production and an increased frequency of monocytes and NK cells [5-8].

Treatments with chemotherapeutic agents and anti-CD20 antibody significantly improved the outcome of CLL patients $[9,10]$. Since the role of BCR as pivotal factor for CLL cells survival [11], numerous studies have focused on treaments inhibiting BTK, a tyrosine kinase downstream BCR. Activation of BTK by a variety of receptors, such as BCR and Fc $\gamma$ Rs, results in proliferation, differentiation and survival of B cells and in maturation, recruitment and activation of neutrophils, monocytes and dendritic cells $[12,13]$. BTK has become a target for anti-B cell leukemia therapies in that it is essential for activation of several pathways involved in CLL cells survival, including AKT, ERK

\footnotetext{
Abbreviations: CLL, chronic lymphocytic leukemia; BTK, Bruton's tyrosine kinase; NE, neutrophils elastase; MPO, myeloperoxidase; LF, lactoferrin; HC, healthy controls; MFI, mean fluorescence intensity; ROS, reactive oxygen species; IL-8, interleukin-8; TNF- $\alpha$, tumor necrosis factor

* Corresponding author.

E-mail address: isabella.quinti@uniroma1.it (I. Quinti).

${ }^{1}$ These authors contributed equally to this work.
} 
and NF-kB pathways $[14,15]$. Given the broad effects of BCR-associated kinases in many tissues, studies using BTK inhibitors need to consider their effects in a rather wide spectrum of tissues and cells, instead of focusing on B cells only [16].

Ibrutinib (PCI-32765) is an orally bioavailable enzyme inhibitor able to irreversibly bind BTK and to suppress numerous pathways involved in tumor progression [17-21].

The infection risks associated to BTK inhibition remained poorly defined. Several reports described opportunistic infections in patients under ibrutinib [22], suggesting possible alterations of the innate immunity $[23,24]$. Our study analyzed in vivo the early and the late effects of ibrutinib-based therapy on neutrophils functions in relapsed/refractory and in naïve CLL patients. We showed a marked reduction of neutrophils killing functions mediated by Fc $\gamma$ Rs in the early phases of treatment and the recovery of these functions after a long-lasting period of treatment.

\section{Material and methods}

\subsection{Patients and healthy controls}

Ten CLL patients ( 6 males and 4 females; age range of 71-82 years; mean age: $74 \pm 4.9$ years) and twelve healthy controls (HC) (7 males and 5 females; age range 26-65 years; mean age $41.6 \pm 13.4$ years) were included in the study. CLL diagnosis was made according to international criteria [25]. Patients with CLL were classified according to the Binet staging system [26,27]. In all CLL patients ibrutinib therapy was administered at the standard dose of $420 \mathrm{mg} /$ daily [28]. No patients were on antibiotic at the time of the present study and in the previous three months. All but one patient (indicated as naïve) were previously treated with other anti-leukemic agents (Supplementary Table 1). Patients included in this study were treated with ibrutinib within the Named Patient Program between April 2014 and January 2015. Before the start of ibrutinib therapy, all but one patients $(9 / 10)$ were previously treated with a median number of three treatments (range 1-6). The median time from last chemo-immunotherapy was six months (range 1-72).

During the first 4-6 weeks of therapy with ibrutinib, all but two patients were on low dose steroids. The reasons for steroid treatment were thrombocytopenia in four patients, Chronic Obstructive Pulmonary Disease (COPD) in two patients and ulcerative colitis in one patient (Supplementary Table 1).

Laboratory informations are provided in Supplementary Table 2. The study was approved by the Ethics Committee of the Sapienza University of Rome and performed in accordance with the Declaration of Helsinki. All participants gave written informed consent prior to inclusion.

\subsection{Blood samples preparation}

Heparinized whole blood samples were collected from twelve HC and ten CLL patients the day before the starting of ibrutinib therapy and during ibrutinib therapy at time points: after forty-eight hours, three weeks and forty months after the initiation of therapy. Total peripheral blood neutrophils count were determined from blood cell counts and white blood cell differentials. For evaluating circulating cells by flow cytometry without the harm of cell loss related to the density gradient centrifugation procedure, peripheral red blood cells were lysed using lysing buffer (Becton Dickinson Biosciences, Franklin Lakes, NJ).

\subsection{Neutrophils oxidative burst}

The leukocyte oxidative burst was analyzed by using the PHAGOBURST assay (Glycotope Biotechnology, Heidelberg, Germany), according to the manufacturers instructions. In addition, we also performed the oxidative burst by using non-opsonized Escherichia coli (E. coli) as control, in order to exclude Fc $\gamma$ Rs-independent phagocytosis. The oxidation leads to fluorescence detected by flow cytometry.

\subsection{Neutrophils elastase, myeloperoxidase and lactoferrin assays}

The NE was quantified by Human neutrophils Elastase Platinum ELISA kit (Affymetrix eBioscience, Cambridge, England), the MPO was quantified by Human Myeloperoxidase ELISA kit (Cohesion Biosciences, London, United Kingdom) and the LF was quantified by Human Lactoferrin ELISA kit (Immunology Consultants Laboratory, Inc, Portland, Oregon) on plasma collected from unstimulated and stimulated blood samples diluted and tested in duplicates according to the manufacturers instructions. Concentrations were expressed as $\mathrm{ng} / \mathrm{ml}$.

\subsection{IL-8 and TNF- $\alpha$ plasma assays}

IL- 8 and TNF- $\alpha$ were measured by using a commercially available ELISA kits (4A Biotech Co, Beijing, China) on plasma samples collected from unstimulated and stimulated blood samples according to the manufacturers' instructions. Concentrations were expressed as $\mathrm{pg} / \mathrm{ml}$.

\subsection{Neutrophils stimulation by Escherichia coli}

One hundred $\mu \mathrm{l}$ of whole blood were added to $20 \mu \mathrm{l}$ of pre-cooled opsonized whole $E$. coli at a concentration of $1-2 \times 10^{9} / \mathrm{ml}$ in order to avoid artifacts due to isolation procedure. Samples were incubated in water bath for $20 \mathrm{~min}$ at $37^{\circ} \mathrm{C}$.

\subsection{Analysis of $C D 11 b$ expression}

The expression of $\mathrm{CD} 11 \mathrm{~b}$ was evaluated on neutrophils from erythrocytes-lysed whole blood samples, performing a staining at $4{ }^{\circ} \mathrm{C}$ for $30 \mathrm{~min}$. Samples were washed, suspended in ice cold PBS and analyzed by a 4-color flow cytometry single platform. Neutrophils were identified by forward scatter (FSC) and side scatter (SSC) characteristics after gating on $\mathrm{CD} 15$ positive events. In parallel, an isotype control ( $\mathrm{IgG}_{1}$, BD) was acquired to exclude autofluorescence background. Results were expressed as geometric Mean Fluorescence Intensity (MFI) within the defined population.

\subsection{Flow cytometry analysis}

Flow cytometric analysis was done with a FACSCalibur instrument (Becton Dickinson Biosciences) using CellQuest (Becton Dickinson Biosciences) and FlowJo (TreeStar, Ashland, Ore) software. The cytometer stability and sensitivity were checked before each acquisition session by using microbeads designed to control the efficiency, the coefficient of variation of scatter and fluorescence signals and the time delay calibration (Nile Red Fluorescent particles and Calibrate APC Beads, all from Becton Dickinson Biosciences). 30.000 events were counted per sample.

\subsection{Statistical analysis}

All data are showed as mean value \pm standard deviation (SD). Data were analyzed using the Mann-Whitney $\mathrm{U}$ test for unpaired two groups or by paired Wilcoxon test. Correlations were calculated by means of linear regression analysis. Data were analyzed with StatView 5.0.1 software (SAS Institute, Cary, NC). A p value equal or less than 0.05 was considered as statistically significant. 


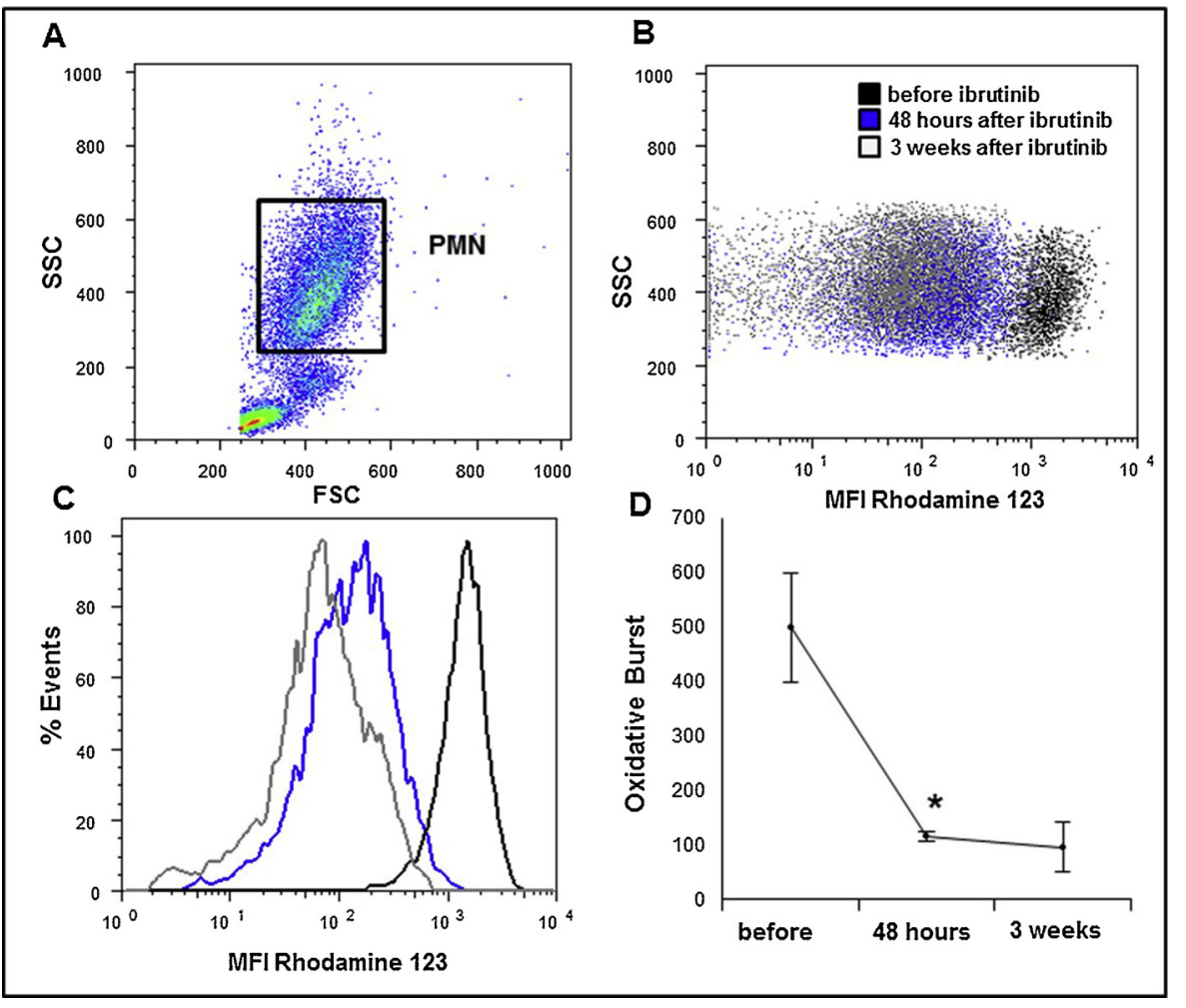

Fig. 1. Ibrutinib therapy inhibits neutrophils $E$. coliinduced oxidative burst. (A) The electronic gate of neutrophils population in a representative CLL patient. (B) The intensity of neutrophils oxidative burst induced by $E$. coli stimulation before, forty-eight hours and three weeks after ibrutinib treatment. (C) Neutrophils oxidative burst markedly decreased fortyeight hours after the first administration of ibrutinib and it remained weak up to three weeks of treatment. Histograms denote the intensity of neutrophils oxidative burst at the different time points analyzed. (D) The neutrophils oxidative burst rapidly fell forty-eight hours after the first ibrutinib administration and it remained weak until three weeks of treatment $(p=0.01)$. Data are shown as MFI \pm SD. Statistical significance is indicated as $\mathrm{p}$ value.

\section{Results}

\subsection{Ibrutinib inhibits neutrophils oxidative burst in the early phases of treatment}

During phagocytosis and in response to soluble agonists, neutrophils generate reactive oxygen species (ROS). This functional response, termed oxidative burst, contributes to host defense, but it can also result in collateral damage of host tissues. Since in vitro studies showed that ibrutinib altered the functions of innate immune cells in CLL [29], we analyzed blood samples stimulated in vitro with $E$. coli from CLL patients who received in vivo the first treatment with ibrutinib in order to investigate the effects on neutrophils functions.

Before ibrutinib CLL patients showed a similar $E$. coli-induced oxidative burst compared to HC $(p=0.4)$. Ibrutinib administration induced a significantly decrease $(p=0.01)$ of oxidative burst already after forty-eight hours of treatment. Moreover, after three weeks, the oxidative burst remained low, indicating a peristent inhibitory effect during the early phases of treatment (Fig. 1, Supplementary Table 3). We confirmed this finding also in newly diagnosed untreated CLL patient (naïve-CLL) who received ibrutinib as a first-line treatment.

\subsection{Ibrutinib therapy impaired $F c \gamma$ Rs-mediated neutrophils degranulation}

Activated neutrophils secrete granules containing a number of serine proteases including (NE), (MPO) and (LF). NE and MPO are stored in primary granules and released during degranulation, exerting an anti-bacterial activity but also collateral damage to host tissue [30,31]. Also LF, stored in secondary granules, exerts antimicrobial, anti-inflammatory and immunomodulatory activities on innate and acquired immune system and anti-carcinogenic activity able to modulate cytokines production [32].

We evaluated the NE, MPO and LF plasma concentration in CLL patients treated with ibrutinib over time. In unstimulated conditions, before ibrutinib administration CLL patients showed a reduced NE $(p=0.003)$, MPO $(p=0.01)$ and LF $(p=0.01)$ plasma levels compared to HC. This difference could be also due to an age effect, since the enrolled HC were younger than patients. Forty-eight hours after the first dose of ibrutinib, NE, MPO and LF plasma levels were still low, while three weeks later MPO $(p=0.02)$ and LF $(p=0.02)$ plasma levels slightly increased (Fig. 2C-F).

In order to estimate the efficiency of Fc $\gamma$ Rs activation during ibrutinib therapy, we evaluated the release of these proteinases after in vitro stimulation with opsonized $E$. coli. In stimulated conditions, before ibrutinib administration NE plasma levels were similar in CLL and HC, while MPO levels were lower in CLL $(p=0.04)$. Forty-eight hours after the first dose of ibrutinib, in stimulated conditions we observed a significant decrease of NE $(p=0.01)$ and MPO $(p=0.04)$ plasma levels. After three weeks of ibrutinib therapy in stimulated conditions, NE $(p=0.01)$ and MPO $(p=0.04)$ plasma levels returned to those observed before treatment (Fig. 2A-D), suggesting a transient inhibition of Fc $\gamma$ Rs-mediated neutrophils degranulation. In stimulated conditions, LF plasma levels remained unchanged at each time point analyzed (Fig. 2E-F). All data are reported in Supplementary Table 3. A similar trend of NE, MPO and LF released in unstimulated and $E$. coli stimulated conditions was also found in the naïve CLL patient treated with ibrutinib as first-line agent.

\subsection{Ibrutinib therapy altered IL-8 plasma levels following $E$. coli stimulation} but not affect TNF- $\alpha$ plasma levels

IL-8 promotes neutrophils recruitment, degranulation and killing activity [33]. IL-8 plasma levels of CLL patients in unstimulated conditions remained unchanged at each time point analyzed. After fortyeight hours from the beginning of therapy, IL-8 levels decreased on plasma stimulated $(p=0.02)$, while after three weeks it returned to the levels observed before ibrutinib treatment $(p=0.03)$. Ibrutinib reduced IL-8 plasma levels after $E$. coli stimulation similarly to that observed for NE and MPO (Fig. 3A-B).

Also TNF- $\alpha$ triggers several neutrophils functions, including oxidative burst [34]. We found a similar TNF- $\alpha$ plasma levels in CLL and HC at each time points analyzed (Fig. 3C). Although we found a dramatic 


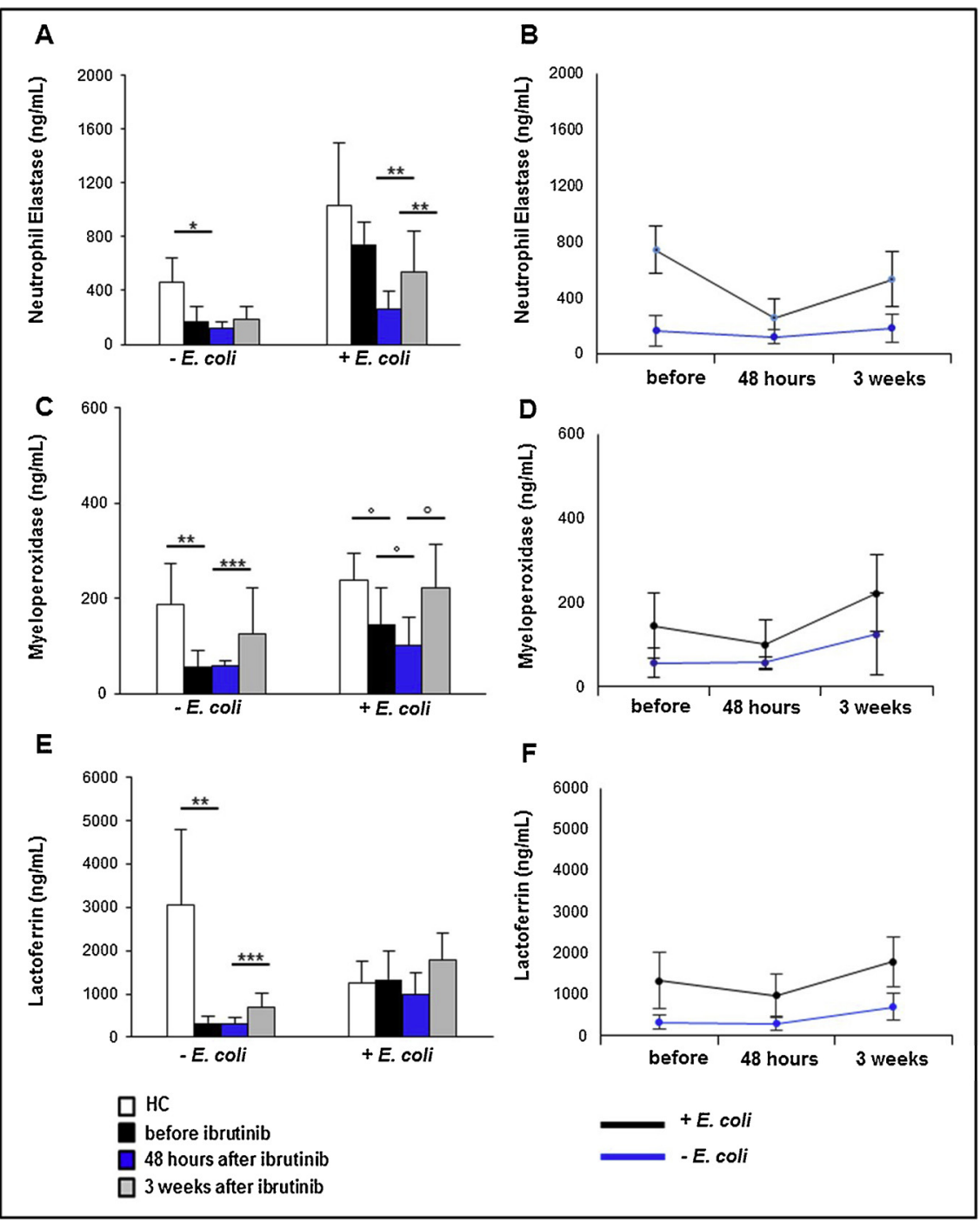

Fig. 2. Ibrutinib therapy affects primary and secondary granules content release. (A) NE plasma concentration in unstimulated conditions was lower in CLL compared to HC ( $\left.{ }^{*} p=0.003\right)$. In $E$. coli stimulated samples, NE plasma concentration was reduced forty-eight hours after the beginning of therapy $(* * p=0.01)$, while it increased after three weeks of treatment ( $* * p=0.01$ ). (B) The trend of NE pasma levels in unstimulated and E. coli-stimulated samples over time. (C) MPO plasma concentration in unstimulated conditions was lower in CLL compared to HC $(* * p=0.01)$, while it increased after three weeks ( $* * * p=0.02$ ). In E. coli stimulated samples, MPO plasma concentration in CLL was reduced compared to HC before treatment $\left({ }^{\circ} p=0.04\right)$ and it further decreased forty-eight hours after the beginning of therapy $\left({ }^{\circ} p=0.04\right)$. MPO plasma concentration increased after three weeks of treatment $\left({ }^{\circ} p=0.04\right)$. (D) The trend of MPO plasma levels in unstimulated and E. coli-stimulated samples overt ime. (E) LF plasma concentration in unstimulated conditions was lower in CLL compared to HC $(* * p=0.01)$, while it increased three weeks later $\left({ }^{* *} p=0.02\right)$. In stimulated samples, LF plasma concentration in CLL was similar to HC at all time points analyzed. (F) The trend of LF plasma levels in unstimulated and E. coli-stimulated samples over time. All data are shown as mean $\pm \mathrm{SD}$. Concentrations are expressed as $\mathrm{ng} / \mathrm{ml}$. Statistical significance is indicated as $\mathrm{p}$ value.

collapse of oxidative burst after ibrutinib administration, this observation suggests the independence of oxidative burst reduction from TNF- $\alpha$. All data are reported in Supplementary Table 3.

\subsection{Ibrutinib therapy did not affect neutrophils $C D 11 b$ expression}

Since CD11b is a neutrophils activation marker [35-37], the analysis of its expression provides information on the activation status of neutrophils. To further analyze the early ibrutinib-mediated effects on neutrophils, we analyzed the CD11b expression on unstimulated and stimulated $\mathrm{CD} 15^{+}$cells. We found a similar CD11b expression in CLL patients and HC for each time point analyzed, suggesting that ibrutinib might not alter the expression of those receptor involved in neutrophils activation (Fig. 4, Supplementary Table 3).

\subsection{Late effects of ibrutinib therapy}

We evaluated the long-lasting therapy effects in a group of six patients survived for more than three years from the beginning of therapy. After forty months of ibrutinib treatment, NE, MPO, LF and IL-8 plasma

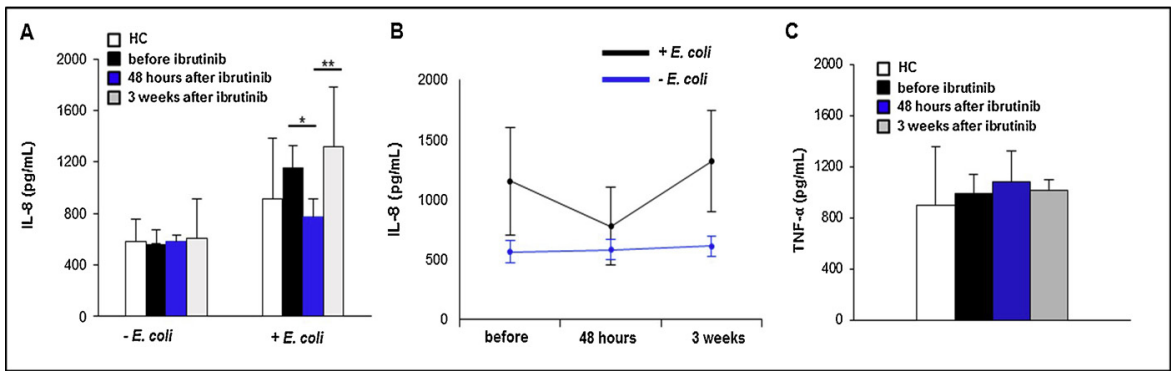

Fig. 3. Ibrutinib therapy alters IL-8 plasma levels following $E$. coli stimulation and it does not affect TNF- $\alpha$ plasma levels. (A) IL-8 plasma concentration in unstimulated conditions in CLL was similar to HC at each time point. In E. coli stimulated samples, IL-8 plasma concentration was reduced forty-eight hours after the beginning of therapy ( ${ }^{*} p=0.02$ ). IL-8 concentration increased after three weeks of treatment ( $* * p=0.03$ ). (B) The trend of IL-8 plasma levels in unstimulated and E. coli-stimulated samples over time. (C) TNF- $\alpha$ plasma concentration in unstimulated samples in CLL was similar to HC at each time point. Data are shown as mean $\pm \mathrm{SD}$. Concentrations are expressed as $\mathrm{pg} / \mathrm{ml}$. Statistical significance is indicated as $p$ value. 


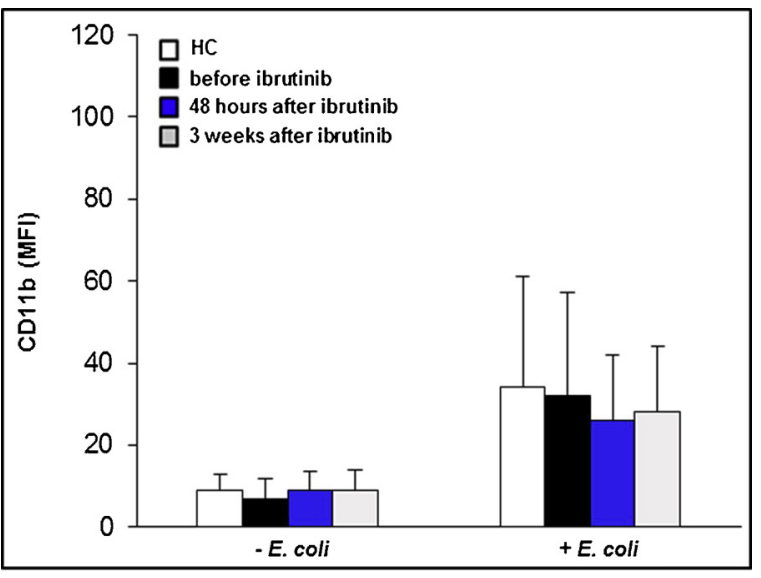

Fig. 4. Ibrutinib therapy does not affect neutrophils CD11b expression. The CD11b surface expression in CLL was similar to HC and it remained unchanged at each time point in unstimulated and in stimulated conditions. Data are shown as MFI \pm SD.

levels and $\mathrm{CD} 11 \mathrm{~b}$ expression in unstimulated conditions increased, reached values similar to those found in HC. The effects of long-lasting therapy on the Fc $\gamma$ Rs-mediated processes induced by opsonized $E$. coli were more variable. Oxidative burst, LF and MPO increased reaching values similar to those found in HC, while NE and IL-8 released remained low. All data are reported in Supplementary Table 3. These results suggest that in the long-term period the effects of BTK pharmacologic inhibition on Fc $\gamma$ Rs-mediated processes were mostly reverted.

\subsection{Correlations between neutrophils count and degranulation}

Before treatment, NE, MPO and LF plasma levels and neutrophils count were positively related (NE: $R=0.76, p=0.018$; MPO: $R=0.72, p=0.03$ ). However, this correlation was lost after ibrutinib administration, showing that the NE and MPO plasma concentrations were not related to the neutrophils count, while LF levels remained related the neutrophils count.

\section{Discussion}

Ibrutinib, a first-in-class inhibitor of BTK that binds covalently the Cys-481 in the ATP-binding domain of the kinase, is effective in the treatment of CLL [15]. BTK is expressed in B cell lineages and in myeloid cells, such as monocytes and neutrophils where it is involved in the Fc $\gamma$ Rs signaling [13]. Therefore, alterations in BTK functions could lead to an impairment of innate immune cells functions, increasing the infectious risk [4]. Nevertheless, we have previously described a wellpreserved neutrophils killing ability in X-linked agammaglobulinemia (XLA) [38], a primary immune deficiency caused by BTK mutations. This observation suggested that the lack of BTK could be compensated by the activation of redundant pathways following the Fc $\gamma$ Rs activation. However, our results in relapsed/refractory CLL patients treated with ibrutinib as single agent showed that the pharmacological inhibition of BTK did not recapitulate the observations made in XLA [38]. Indeed, we observed a dramatic reduction of neutrophils oxidative burst after the first forty-eight hours of ibrutinib therapy. The oxidative burst remained at low level up to three weeks of therapy indicating a persistent inhibition. The same effect was observed also in a naïve CLL patient, confirming the inhibition of neutrophils functions. Our data are in line with Stadler et al [39] that showed a reduced TREM-1-mediated oxidative burst in patients with recurrent B-cell non-Hodgkin lymphomas receiving ibrutinib. Differently from the lack of BTK in XLA, the impairment of Fc $\gamma$ Rs-mediated processes induced by ibrutinib might be

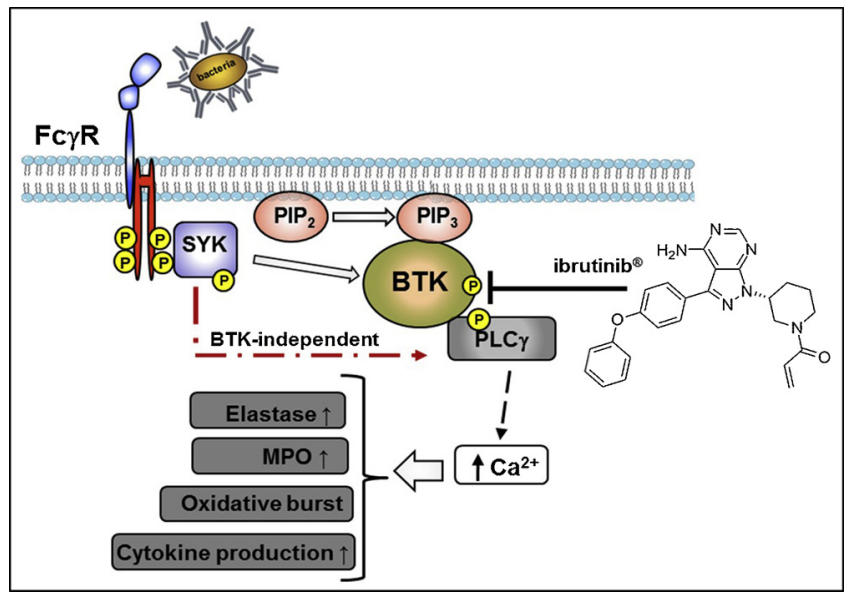

Fig. 5. Schematic representation of ibrutinib blockage on Fc $\gamma$ Rs signaling pathway. Opsonized bacteria engage Fc $\gamma$ Rs and trigger their activation. BTK activation induces an increase of intracellular calcium concentration leading to oxidative burst, degranulation and cytokine production. Ibrutinib, by inhibiting BTK, causes an impairment of cellular responses after Fc $\gamma$ Rs activation. SYK is also directly involved in PLC $\gamma$ activation. The initial inhibitory effect exerted by ibrutinib might be compensated over time by Btk-independent pathways that restore neutrophil functions.

due to an initial inefficiency of BTK-independent pathways (Fig. 5). In addition, since ibrutinib inhibits several other kinases, the off-target effects on non-BTK Tec family proteins may alter the neutrophils functions [40]. Consistently, it has been observed that acalabrutinib, a more specific inhibitor of BTK, has a limited impact on Fc $\gamma$ Rs-mediated processes [41], even if in the Ascendent trial presented at the 2019 EHA meeting, infections were also reported in a relevant proportion of patients treated with acalabrutinib as a single agent.

The reduced phagocytosis-mediated ROS production observed in our study is in line with Ferrarini et al [42] and Da Roit et al [43] that demonstrated how ibrutinib reduced monocytes and neutrophils phagocytosis. On the contrary, when the phagocytic step was avoided using PMA (phorbol12-myristate 13-acetate), a potent NADPH oxidase inducer that interact directly with protein kinase $\mathrm{C}$ (PKC), the inhibition was no longer evident [42].

Similarly to the oxidative burst, the release of NE and MPO induced by opsonized $E$. coli appeared reduced after forty-eight hours of therapy, confirming that ibrutinib exerted a depressive effect on Fc $\gamma$ Rsmediated processes. Moreover, the amount of NE and MPO did not correlate with neutrophil counts suggesting that all these effects were not due to a reduction of neutrophil counts. Our results showed that ibrutinib induced a rapid reduction of Fc $\gamma$ Rs-mediated IL- 8 production, a cytokine responsible of a series of physiological responses such as migration, phagocytosis, increase of intracellular $\mathrm{Ca}^{2+}$, degranulation and ROS production [44,45]. ROS, in turn, increase IL-8 secretion leading to a worsening of oxidative stress, therefore ibrutinib might impaired this positive feedback. However, after three weeks of ibrutinib therapy, the IL-8, NE and MPO release was restored suggesting an increased efficiency of alternative pathways that bypass BTK inhibition (Fig. 5). In order to verify the late influence of therapy on neutrophils functions, we evaluated the ibrutinib effects in six CLL patients survived for more than three years. Most Fc $\gamma$ Rs-mediated processes, as the oxidative burst, MPO and LF degranulation were completely recovered. Thus, it seems that patients could receive extended treatment with minimal negative effects on innate immunity.

\section{Conclusions}

Taken together our data suggest that during the first phases of treatment, the inhibition of BTK suppresses Fc $\gamma$ Rs-mediated neutrophils 
functions, potentially in a clinically relevant way. This neutrophils impairment might represent an additional infectious risk in CLL patients receiving ibrutinib beyond the impairment of B-cell responses. Anyway, during the late fases of treatment we observed an improvement in neutrophils functions, suggesting a reduction of infectious risk. Our results are consistent with the observation that infectious complications appear to occur more frequently during the early part of treatment and decline over time during ibrutinib therapy [27,46,47]. Reasons for the decrease in infections morbidity over time are potentially related to several factors and the improvement of neutrophilic activity can be one of them.

Considering that ibrutinib is a potent inhibitor of key pathways in neutrophils activities, further studies on a greater cohort of patients are needed to determine the immunologic consequences of prolonged BTK inhibition, since that a limitation of this study was the low number of CLL patients recruited.

\section{Declaration of Competing Interest}

All the authors have no interests to declare.

\section{Acknowledgments}

This study was funded by Progetti Ateneo 2018 and supported by the Jeffrey Modell Foundation.

\section{Appendix A. Supplementary data}

Supplementary material related to this article can be found, in the online version, at doi:https://doi.org/10.1016/j.leukres.2019.106233.

\section{References}

[1] T.J. Kipps, F.K. Stevenson, C.J. Wu, C.M. Croce, G. Packham, W.G. Wierda, S. O'Brien, J. Gribben, K. Rai, Chronic lymphocytic leukaemia, Nat. Rev. Dis Primers 3 (2017) 16096, https://doi.org/10.1038/nrdp.2016.96.

[2] G. Fabbri, R. Dalla-Favera, The molecular pathogenesis of chronic lymphocytic leukaemia, Nat. Rev. Cancer 16 (2016) 145-162, https://doi.org/10.1038/nrc. 2016.8.

[3] P. Giannoni, G. Cutrona, D. Totero, Survival and immunosuppression induced by hepatocyte growth factor in chronic lymphocytic leukemia, Curr. Mol. Med. 17 (2017) 24-33, https://doi.org/10.2174/1566524017666170220095838.

[4] V.A. Morrison, Infectious complications of chronic lymphocytic leukaemia: pathogenesis, spectrum of infection, preventive approaches, Best Pract. Res. Clin. Haematol. 23 (2010) 145-153, https://doi.org/10.1016/j.beha.2009.12.004.

[5] G. Füst, Z. Miszlay, E. Czink, L. Varga, K. Pálóczi, G. Szegedi, S.R. Hollán, C1 and C4 abnormalities in chronic lymphocytic leukaemia and their significance, Immunol. Lett. 14 (1987) 255-259, https://doi.org/10.1016/0165-2478(87)90110-6.

[6] D.P. Kontoyiannis, S.P. Georgiadou, W.G. Wierda, S. Wright, N.D. Albert, A. Ferrajoli, M. Keating, R.E. Lewis, Impaired bactericidal but not fungicidal activity of polymorphonuclear neutrophils in patients with chronic lymphocytic leukemia, Leuk. Lymphoma 54 (2013) 1730-1733, https://doi.org/10.3109/ 10428194.2012.750723.

[7] D. Flieger, H.W. Ziegler-Heitbrock, Abnormal blood monocytes in chronic lymphocytic leukemia, Cancer Res. 48 (1988) 4812-4816.

[8] J.A. Burger, J.G. Gribben, The microenvironment in chronic lymphocytic leukemia (CLL) and other B cell malignancies: insight into disease biology and new targeted therapies, Semin. Cancer Biol. 24 (2014) 71-81, https://doi.org/10.1016/j. semcancer.2013.08.011.

[9] S.H. Lim, S.A. Beers, R.R. French, P.W.M. Johnson, M.J. Glennie, M.S. Cragg, AntiCD20 monoclonal antibodies: historical and future perspectives, Haematologica 95 (2010) 135-143, https://doi.org/10.3324/haematol.2008.001628.

[10] C. Bagacean, M. Zdrenghea, A. Tempescul, V. Cristea, Y. Renaudineau, Anti-CD20 monoclonal antibodies in chronic lymphocytic leukemia: from uncertainties to promises, Immunotherapy 8 (2016) 569-581, https://doi.org/10.2217/imt-20150015 .

[11] A. Wiestner, BCR pathway inhibition as therapy for chronic lymphocytic leukemia and lymphoplasmacytic lymphoma, Hematol. Am. Soc. Hematol. Educ. Program 5 (2014) 125-134, https://doi.org/10.1182/asheducation-2014.1.125.

[12] J.A. Woyach, E. Bojnik, A.S. Ruppert, M.R. Stefanovski, V.M. Goettl, K.A. Smucker, L.L. Smith, J.A. Dubovsky, W.H. Towns, J. MacMurray, B.K. Harrington, M.E. Davis, S. Gobessi, L. Laurenti, B.Y. Chang, J.J. Buggy, D.G. Efremov, J.C. Byrd, A.J. Johnson, Bruton's tyrosine kinase (BTK) function is important to the development and expansion of chronic lymphocytic leukemia (CLL), Blood 123 (8) (2014) 1207-1213, https://doi.org/10.1182/blood-2013-07-515361.
[13] A.N.R. Weber, Z. Bittner, X. Liu, T.M. Dang, M.P. Radsak, C. Brunner, Bruton's tyrosine kinase: an emerging key player in innate immunity, Front. Immunol. 8 (2017) 1454, https://doi.org/10.3389/fimmu.2017.01454.

[14] A.F. Muggen, S.P. Singh, R.W. Hendriks, A.W. Langerak, Targeting signaling pathways in chronic lymphocytic leukemia, Curr. Cancer Drug Targets 16 (2016) 669-688, https://doi.org/10.2174/1568009616666160408145623.

[15] R.W. Hendriks, S. Yuvaraj, L.P. Kil, Targeting Bruton's tyrosine kinase in B cell malignancies, Nat. Rev. Cancer 14 (2014) 219-232, https://doi.org/10.1038/ nrc3702.

[16] P.H. Nguyen, E. Niesen, M. Hallek, New roles for B cell receptor associated kinases: when the B cell is not the target, Leukemia 33 (2019) 576-587, https://doi.org/10. 1038/s41375-018-0366-8.

[17] S.E. Herman, A.L. Gordon, E. Hertlein, A. Ramanunni, X. Zhang, S. Jaglowski, J. Flynn, J. Jones, K.A. Blum, J.J. Buggy, A. Hamdy, A.J. Johnson, J.C. Byrd, Bruton tyrosine kinase represents a promising therapeutic target for treatment of chronic lymphocytic leukemia and is effectively targeted by PCI-32765, Blood 117 (23) (2011) 6287-6296, https://doi.org/10.1182/blood-2011-01-328484.

[18] L.A. Honigberg, A.M. Smith, M. Sirisawad, E. Verner, D. Loury, B. Chang, S. Li, Z. Pan, D.H. Thamm, R.A. Miller, J.J. Buggy, The Bruton tyrosine kinase inhibitor PCI-32765 blocks B-cell activation and is efficacious in models of autoimmune disease and B-cell malignancy, Proc. Natl. Acad. Sci. U. S. A. 107 (29) (2010) 13075-13080, https://doi.org/10.1073/pnas.1004594107.

[19] R.Z. Mustafa, S.E.M. Herman, J. Jones, J. Gyamfi, M. Farooqui, A. Wiestner, Ibrutinib inhibits B-cell adhesion and causes an efflux of chronic lymphocytic leukemia cells from the tissue microenvironment into the blood leading to a transient treatment-induced lymphocytosis, Blood 122 (2013) 674.

[20] S.E. Herman, R.Z. Mustafa, J.A. Gyamfi, S. Pittaluga, S. Chang, B. Chang, M. Farooqui, A. Wiestner, Ibrutinib inhibits BCR and NF-kB signaling and reduces tumor proliferation in tissue-resident cells of patients with CLL, Blood 123 (2014) 3286-3295, https://doi.org/10.1182/blood-2014-02-548610.

[21] S. Cheng, J. Ma, A. Guo, P. Lu, J.P. Leonard, M. Coleman, M. Liu, J.J. Buggy, R.R. Furman, Y.L. Wang, BTK inhibition targets in vivo CLL proliferation through its effects on B-cell receptor signaling activity, Leukemia 28 (2014) 649-657, https:// doi.org/10.1038/leu.2013.358.

[22] N.D. Diaco, B. Strohdach, A.L. Falkowski, N. Hainc, P. Brunner, J. Rutishauser, L. Jost, P.E. Tarr, Psoas abscess due to Mycobacterium avium in a patient with chronic lymphocytic leukemia - case report and review, J. Clin. Med. 8 (2019), https://doi.org/10.3390/jcm8020216 pii: E216.

[23] T. Varughese, Y. Taur, N. Cohen, M.L. Palomba, S.K. Seo, T.M. Hohl, G. RedelmanSidi, Serious infections in patients receiving ibrutinib for treatment of lymphoid cancer, Clin. Infect. Dis. 67 (2018) 687-692, https://doi.org/10.1093/cid/ciy175

[24] B.F. Tillman, J.M. Pauff, G. Satyanarayana, M. Talbott, J.L. Warner, Systematic review of infectious events with the Bruton tyrosine kinase inhibitor ibrutinib in the treatment of hematologic malignancies, Eur. J. Haematol. 100 (2018) 325-334, https://doi.org/10.1111/ejh.13020.

[25] M. Hallek, B.D. Cheson, D. Catovsky, F. Caligaris-Cappio, G. Dighiero, H. Döhner, P. Hillmen, M. Keating, E. Montserrat, N. Chiorazzi, S. Stilgenbauer, K.R. Rai, J.C. Byrd, B. Eichhorst, S. O’Brien, T. Robak, J.F. Seymour, T.J. Kipps, iwCLL guidelines for diagnosis, indications for treatment, response assessment, and supportive management of CLL, Blood 131 (2018) 2745-2760, https://doi.org/10. 1182/blood-2017-09-806398.

[26] K.R. Rai, A. Sawitsky, E.P. Cronkite, A.D. Chanana, R.N. Levy, B.S. Pasternack, Clinical staging of chronic lymphocytic leukemia, Blood 46 (1975) 219-234, https://doi.org/10.1182/blood-2016-08-737650.

[27] J.L. Binet, A. Auquier, G. Dighiero, C. Chastang, H. Piguet, J. Goasguen, G. Vaugier, G. Potron, P. Colona, F. Oberling, M. Thomas, G. Tchernia, C. Jacquillat, P. Boivin, C. Lesty, M.T. Duault, M. Monconduit, S. Belabbes, F. Gremy, A new prognostic classification of chronic lymphocytic leukemia derived from a multivariate survival analysis, Cancer 48 (1981) 198-206.

[28] J.C. Byrd, R.R. Furman, S.E. Coutre, I.W. Flinn, J.A. Burger, K.A. Blum, B. Grant, J.P. Sharman, M. Coleman, W.G. Wierda, J.A. Jones, W. Zhao, N.A. Heerema, A.J. Johnson, J. Sukbuntherng, B.Y. Chang, F. Clow, E. Hedrick, J.J. Buggy, D.F. James, S. O'Brien, Targeting BTK with ibrutinib in relapsed chronic lymphocytic leukemia, N. Engl. J. Med. 369 (2013) 32-42 Erratum in: N Engl J Med. 370 (2014) 786. https://doi.org/10.1056/NEJMoa1215637.

[29] S. Fiorcari, R. Maffei, V. Audrito, S. Martinelli, E. Ten Hacken, P. Zucchini, G. Grisendi, L. Potenza, M. Luppi, J.A. Burger, S. Deaglio, R. Marasca, Ibrutinib modifies the function of monocyte/macrophage population in chronic lymphocytic leukemia, Oncotarget 7 (2016) 65968-65981, https://doi.org/10.18632/ oncotarget.11782.

[30] C.K. Mårdh, J. Root, M. Uddin, K. Stenvall, A. Malmgren, K. Karabelas, M. Thomas, Targets of neutrophil influx and weaponry: therapeutic opportunities for chronic obstructive airway disease, J. Immunol. Res. 2017 (2017) 5273201, , https://doi. org/10.1155/2017/5273201.

[31] P. Lacy, Mechanisms of degranulation in neutrophils, Allergy Asthma Clin. Immunol. 2 (2006) 98-108, https://doi.org/10.1186/1710-1492-2-3-98.

[32] S.A. González-Chávez, S. Arévalo-Gallegos, Q. Rascón-Cruz, Lactoferrin: structure, function and applications, Int. J. Antimicrob. Agents 33 (2009) 301, https://doi. org /10.1186/1710-1492-2-3-98.

[33] H. Ha, B. Debnath, N. Neamati, Role of the CXCL8-CXCR1/2 axis in cancer and inflammatory diseases, Theranostics 7 (2017) 1543-1588, https://doi.org/10. $7150 /$ thno. 15625.

[34] T. Jevtovic-Stoimenov, T. Cvetkovic, M. Despotovic, J. Basic, J. Cvetkovic, G. Marjanovic, D. Pavlovic, The influence of TNF alpha -308 G/A polymorphism on oxidative stress in patients with chronic lymphocytic leukemia, Leuk. Res. 54 (2017) 66-72, https://doi.org/10.1016/j.leukres.2017.01.018. 
[35] L. Fossati-Jimack, G.S. Ling, A. Cortini, M. Szajna, T.H. Malik, J.U. McDonald, M.C. Pickering, H.T. Cook, P.R. Taylor, M. Botto, Phagocytosis is the main CR3mediated function affected by the lupus-associated variant of $\mathrm{CD} 11 \mathrm{~b}$ in human myeloid cells, PLoS One 8 (2013) e57082, , https://doi.org/10.1371/journal.pone. 0057082.

[36] J. Lim, N.A. Hotchin, Signalling mechanism of the leukocyte integrin $\alpha \mathrm{M} \beta 2$ : current and future perspectives, Biol. Cell 104 (2012) 631-640, https://doi.org/10.1111/ boc. 201200013.

[37] M. Siddiqi, Z.C. Garcia, D.S. Stein, T.N. Denny, Z. Spolarics, Relationship between oxidative burst activity and CD11b expression in neutrophils and monocytes from healthy individuals: effects of race and gender, Cytometry 46 (2001) 243-246.

[38] F.M. Cavaliere, A. Prezzo, C. Bilotta, M. Iacobini, I. Quinti, The lack of BTK does not impair monocytes and polymorphonuclear cells functions in X-linked agammaglobulinemia under treatment with intravenous immunoglobulin replacement, PLoS One 12 (2017) e0175961, , https://doi.org/10.1371/journal.pone.0175961.

[39] N. Stadler, A. Hasibeder, P.A. Lopez, D. Teschner, A. Desuki, O. Kriege, A.N.R. Weber, C. Schulz, C. Michel, G. Heß, M.P. Radsak, The Bruton tyrosine kinase inhibitor ibrutinib abrogates triggering receptor on myeloid cells 1-mediated neutrophil activation, Haematologica 102 (2017) e191-e194, https://doi.org/10. 3324/haematol.2016.152017.

[40] A. Aw, J.R. Brown, Current status of Bruton's tyrosine kinase inhibitor development and use in B-cell malignancies, Drugs Aging 34 (2017) 509-527, https://doi.org/ 10.1007/s40266-017-0468-4.

[41] K.R. VanDerMeid, M.R. Elliott, A.M. Baran, P.M. Barr, C.C. Chu, C.S. Zent, Cellular cytotoxicity of next-generation CD20 monoclonal antibodies, Cancer Immunol. Res.
6 (2018) 1150-1160, https://doi.org/10.1158/2326-6066.

[42] I. Ferrarini, A. Rigo, A. Montresor, C. Laudanna, F. Vinante, Monocyte-to-macrophage switch reversibly impaired by Ibrutinib, Oncotarget 10 (2019) 1943-1956, https://doi.org/10.18632/oncotarget.26744.

[43] F. Da Roit, P.J. Engelberts, R.P. Taylor, E.C. Breij, G. Gritti, A. Rambaldi, M. Introna, P.W. Parren, F.J. Beurskens, J. Golay, Ibrutinib interferes with the cellmediated anti-tumor activities of therapeutic CD20 antibodies: implications for combination therapy, Haematologica 100 (2015) 77-86, https://doi.org/10.3324/ haematol.2014.107011.

[44] N. Borregaard, Neutrophils, from marrow to microbes, Immunity 33 (2010) 657-670, https://doi.org/10.1016/j.immuni.2010.11.011.

[45] G. Ricevuti, Host tissue damage by phagocytes, Ann. NY Acad. Sci. 832 (1997) 426-448, https://doi.org/10.1111/j.1749-6632.1997.tb46269.

[46] C. Sun, X. Tian, Y.S. Lee, S. Gunti, A. Lipsky, S.E.M. Herman, D. Salem, M. StetlerStevenson, C. Yuan, L. Kardava, S. Moir, I. Maric, J. Valdez, S. Soto, G.E. Marti, M.Z. Farooqui, A.L. Notkins, A. Wiestner, G. Aue, Partial reconstitution of humoral immunity and fewer infections in patients with chronic lymphocytic leukemia treated with ibrutinib, Blood 126 (2015) 2213-2219, https://doi.org/10.1182/ blood-2015-04-639203.

[47] J.C. Byrd, R.R. Furman, S.E. Coutre, J.A. Burger, K.A. Blum, M. Coleman, W.G. Wierda, J.A. Jones, W. Zhao, N.A. Heerema, A.J. Johnson, Y. Shaw, E. Bilotti, C. Zhou, D.F. James, S. O’Brien, Three-year follow-up of treatment-naïve and previously treated patients with CLL and SLL receiving single-agent ibrutinib, Blood 125 (2015) 2497-2506, https://doi.org/10.1182/blood-2014-10-606038. 